\title{
(6) OPEN ACCESS \\ Effectiveness of combined macular buckle under direct vision and vitrectomy with ILM peeling in refractory macular hole retinal detachment with extreme high axial myopia: a 24-month comparative study
}

\author{
Jin Ma, Honghui Li, Xiaohu Ding, Silvia Tanumiharjo, Lin Lu
}

Zhongshan Ophthalmic Center, Sun Yat-sen University, State Key Laboratory of Ophthalmology, Guangzhou, People's Republic of China

\section{Correspondence to}

Professor Lin Lu, Zhongshan Ophthalmic Center, Sun Yat-sen University, State Key Laboratory of Ophthalmology, 510060, No. 54 Xianlie road, Guangzhou, 510060, People's Republic of China; drlulin@126.com

Received 30 December 2016 Revised 3 February 2017 Accepted 10 February 2017 Published Online First 14 March 2017

\section{CrossMark}

To cite: Ma J, Li H, Ding $X$ et al. Br J Ophthalmol 2017;101:1386-1394.

\section{ABSTRACT}

Purpose To evaluate the efficacy of a combined macular buckle under direct vision and 23-gauge pars plana vitrectomy (PPV) with internal limiting membrane (ILM) peeling in refractory macular hole retinal detachment (MHRD) with extreme high axial myopia. Design Prospective, randomised controlled study. Participants The study included 98 eyes of 98 patients of MHRD with extreme high axial (>30 mm) myopia.

Intervention Patients were randomly assigned to undergo PPV with ILM peeling (group 1, $n=52$ ) or PPV with ILM peeling combined with macular buckle under direct vision (group 2, $\mathrm{n}=46$ ).

Main outcome measures Complete ocular examination included best-corrected visual acuity (BCVA) (LogMAR), applanation tonometry, optical biometry, slit-lamp biomicroscopy, colour fundus photography, ultrasound examination and optical coherence tomography at baseline and every follow-up visit.

Results Initial retinal reattachment rate was significantly higher in group 2 than in group 1 at 12 month postoperatively $\left(\chi^{2}\right.$ test, $\left.p=0.020\right)$. Macular hole closure rate in group 2 was significantly higher than that in group 1 at 3, 12, 18 and 24 months postoperatively (Fisher's exact test, $\mathrm{p}<0.05$ ). In initial retinal reattachment cases, the mean BCVA decreased significantly in group 2 than in group 1 at 3 months postoperatively (Wilcoxon matched pairs signed rank test, $p=0.036$ ), and had increased significantly in group 2 than in group 1 since 6 months postoperatively (Wilcoxon matched pairs signed rank test, $p<0.05$ ). Mean axial lengths in group 2 were significantly shorter than that of group 1 at each follow-up time point (Wilcoxon matched pairs signed rank test, $p<0.05$ ). Conclusions Combined macular buckle under direct vision and PPV with ILM peeling is more effective in treatment of MHRD with extreme high axial (>30 mm) myopia.

\section{INTRODUCTION}

Retinal detachment resulting from a macular hole occurs most commonly in high myopic eyes. ${ }^{12}$ The greater the axial length, the greater the risk of developing a posterior retinal detachment, due to the inability of the retina to adapt to the progressive axial elongation in eyes with high myopia and posterior staphyloma. ${ }^{3}$ Morita et $a l^{4}$ found incidences of macular hole retinal detachment
(MHRD) of $97.6 \%$ in myopia over $-8.25 \mathrm{D}$ (dioptres), $67.7 \%$ in myopia between -8.0 and $-3.25 \mathrm{D}$, and $1.1 \%$ in myopia under $-3.0 \mathrm{D}$, with a higher percentage in eyes with posterior staphyloma (96\%) than eyes without (8.2\%). For many years, pars plana vitrectomy (PPV) with gas or silicone oil tamponade, internal limiting membrane (ILM) peeling with or without epiretinal membrane peeling has been preferred as the first choice in the treatment for MHRD in highly myopic eyes. ${ }^{5}$ But recent case series indicated that PPV and ILM peeling achieve a poor anatomic success rate and high recurrence rate postoperatively. ${ }^{6-8}$ This is because retinal traction cannot be completely eliminated by means of vitreoretinal surgery alone (PPV and ILM peeling) since some of the components causing retinal stretching, such as vascular traction and posterior staphyloma, are still present. ${ }^{9}{ }^{10}$ In order to solve the problem, a variety of episcleral surgeries such as posterior scleral reinforcement and macular buckle are designed to change the concavity of the posterior part of the eye into a flatter or even convex shape, and release the inverse traction caused by the posterior staphyloma. ${ }^{6}$ However, these surgical procedures still have some disadvantages including inability to check the exact position of buckle intraoperatively, and lack of buckling strength control. ${ }^{6} 11$ To overcome these difficulties, some new methods are designed. ${ }^{12-14}$ Parolini ${ }^{15}$ described a L-shaped buckle made of silicone sponge containing a titanium stent to indent the macula. Fujikawa ${ }^{12}$ used the scleral imbrication technique made by sutures on the temporal side to produce shortening of axial length and flattening of the posterior eye wall including the posterior staphyloma. Mura et $a l^{16}$ positioned a T-shaped macular buckling made of solid silicone under the macular. Nevertheless, all these approaches still could not be performed under direct vision, and even cause some complications at the posterior pole (such as subretinal haemorrhages) associated with suture at the back of the eye globe, which could attenuated the effect of the surgery. Thus, MHRD is really a refractory disease with poor visual prognosis. ${ }^{17}$ Despite all the above interventions, reopening of the macular hole and recurrent retinal detachment may still develop, especially for extreme high axial myopia. Previous study demonstrate that axial length of $30.0 \mathrm{~mm}$ or more may increase the risk of anatomic failure of macular 
hole surgery. 9101819 Therefore, we design a combined surgery of macular buckle under direct vision and PPV with ILM peeling for cases of MHRD with extreme high axial (>30 mm) myopia in a prospective, 24-month comparative cohort study.

\section{METHODS}

This prospective, randomised controlled study was conducted according to the guidelines of the Declaration of Helsinki and approved by the institutional ethics committee of Zhongshan Ophthalmic Center, Sun Yat-Sen University. Patients with extreme high axial myopia with MHRD were recruited in Zhongshan Ophthalmic Center from November 2012 to September 2014. Written informed consent for participation was obtained from all patients. The patients were operated on by a same surgeon (Jin Ma). The extreme high axial myopia was defined as an eye with an axial length of $>30 \mathrm{~mm}$. The MHRD was assessed by indirect ophthalmoscope, ultrasonography and fundus photography. Eligible patients gave informed consent and had agreed to attend follow-up visits for a minimum of 24 months after the surgery. Exclusion criteria included MHRD associated with peripheral retinal break, idiopathic macular hole without posterior staphyloma, traumatic macular hole, retinal break or detachment secondary to vitreoretinal exudations or proliferations. Patients with a history of systemic diseases such as diabetes mellitus were also excluded. Eyes were excluded if they had pre-existing ocular diseases or a history of retinal surgery, especially PPV and scleral buckle, except for cataract surgery, or the other eye belonging to the WHO categories of blindness. A computer-generated list of random numbers was used to split the recruited patients into two groups: group 1 underwent 23-gauge PPV with ILM peeling, and group 2 underwent 23-gauge PPV with ILM peeling combined with macular buckle under direct vision. Only one eye per patient was included in our study.

\section{Surgical procedures}

The procedure was carried out under general anaesthesia. In group 2 with combined macular buckle and PPV with ILM peeling surgery, the silicone buckling element was made of a piece of solid tire $(6 \times 15 \mathrm{~mm})$ sewn with an encircling silicone band ( $3 \mathrm{~mm}$ width) (figure $1 \mathrm{~A})$. First, four rectus muscles were isolated, and silicone buckle was positioned and passed underneath each of the four rectus muscles and the inferior oblique muscle (figure 1B). After that, a 23 -gauge 3-port PPV with separation of the vitreoretinal adhesion between the posterior vitreous membrane and the retina was performed in all patients, using the Constellation Vitrectomy System (Alcon Laboratories). Triamcinolone acetonide was used intraoperatively to facilitate visualisation of the vitreous and posterior hyaloids in all patients. ILM around the macular hole was peeled off until to the edge of the staphyloma successfully with forceps after staining with triamcinolone acetonide. Finally, the silicone tire was

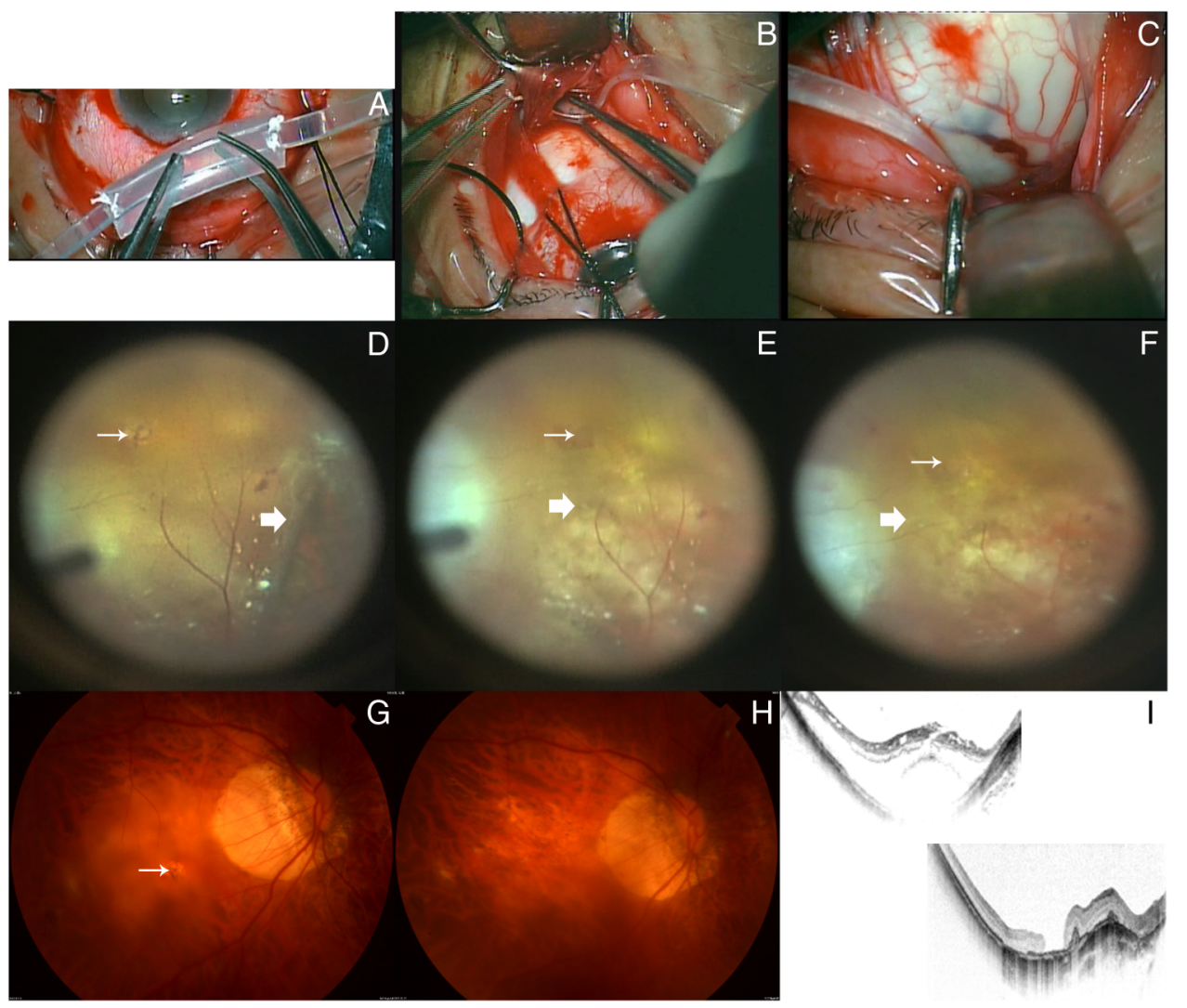

Figure 1 Surgical procedure of macular buckle and postoperative outcome in a same case. (A) The buckling element was made of a piece of silicone tire $(6 \times 15 \mathrm{~mm})$ sewn with an encircling silicone band (3 mm width). (B) Buckle was passed underneath the inferior oblique muscle. (C) Congested and tortuous vortex veins during surgical process. (D) After pars plana vitrectomy (PPV) and internal limiting membrane (ILM) peeling around macular hole (thin arrow), the buckle indentation initially located at equator (thick arrow). (E) Episcleral silicone (thick arrow) tire was moved to the posterior pole of the eyeball gradually under direct vision. (F) Location of buckle was adjusted under direct vision until macular indentation (thick arrow) was under macular hole (thin arrow). (G) Preoperative fundus photograph with macular hole retinal detachment (MHRD) (thin arrow). (H) 24 months postoperative fundus photograph with retina reattachment. I. Preoperative (upper) and 24 months postoperative (down) optical coherence tomography examination with unclosed macular hole and flattened staphyloma. 
moved to the posterior pole of the eyeball gradually and positioned under the macular hole at staphyloma area, which guided under direct vision by endoillumination of light pipe (figure 1D-F). Two free end of encircling band was jointed with a silicone sleeve and sewn onto the nasal quadrant of the globe (figure 2). After the band was tightened, the buckling tension was adjusted under direct vision to flatten the posterior staphyloma until the buckling crest conformed to or a little higher than the arc-shaped posterior scleral wall. Apical flattening of a posterior staphyloma could be seen. During the process, it was necessary to stop tightening the encircling band to avoid the overcompression on the eyeball on the condition of congested and tortuous vortex veins appearance. Then flattening of the retina was achieved with fluid/air exchange, and $12 \%$ perfluoropropane gas $\left(\mathrm{C}_{3} \mathrm{~F}_{8}\right)$ tamponade. In group 1 , all cases underwent PPV with ILM peeling alone in the same manner. All patients kept staying in prone positioning for at least 1 week postoperatively. Phacoemulsification on all phakic eyes with intraocular lens implantation were performed prior to MHRD surgery, for patients were all elderly and expected to have cataract after the PPV and gas tamponade surgery.

\section{Evaluations}

At the time of recruitment, all patients had undergone comprehensive ophthalmological examinations, including best-corrected visual acuity (BCVA) (LogMAR) using Early Treatment Diabetic Retinopathy Study (ETDRS) chart, axial length using optical biometry (signal-to-noise ratio: >100) (IOL Master 500; Carl Zeiss Meditec AG, Jane, Germany), macular hole (Figure 1G) and retina status using SD-optical coherence tomography (Heidelberg Engineering, Heidelberg, Germany) as well as ultrasonography, intraocular pressure (IOP) using applanation tonometry, slit-lamp and indirect ophthalmoscope examination. Patients with failure to read any letters were tested using counting fingers, hand movements or light perception. BCVA (LogMAR) of counting fingers and hand movements were arbitrarily assigned

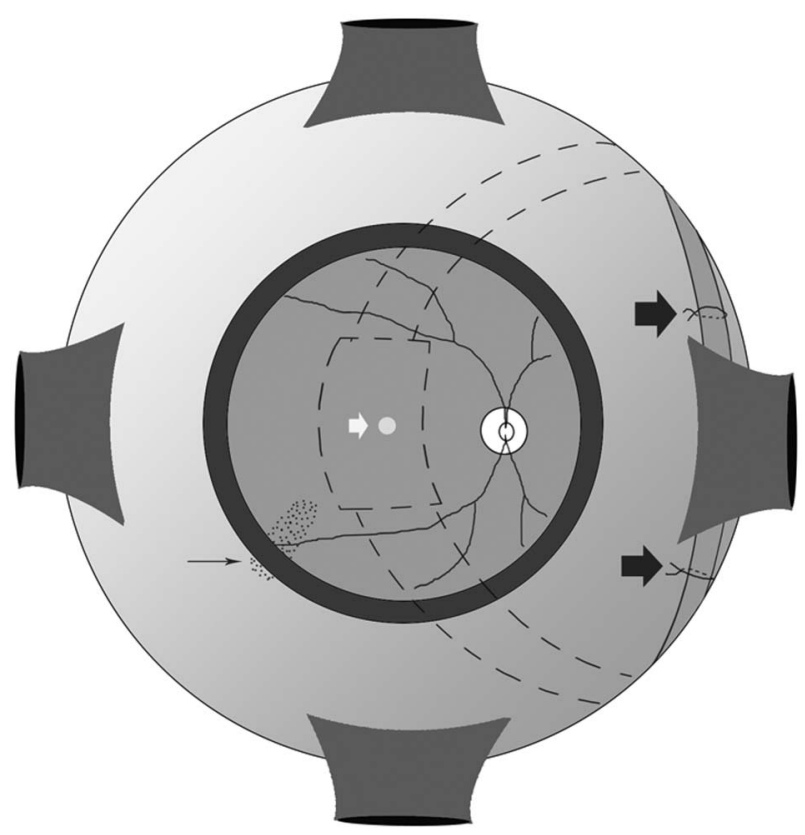

Figure 2 The schematic for surgical procedure. The encircling band was fixed and sutured on the sclera of nasal quadrant (thick arrows). The silicone band and buckle (dashed line) were located on the nasal side of inferior oblique muscle termination (thin arrow). The white arrow points to the macular hole. an equivalent of 2.3 and 2.6 LogMAR units, respectively, ${ }^{20}$ and of light perception and no light perception were assigned an equivalent of LogMAR 3.0 and LogMAR 4.0, respectively. ${ }^{21}$ The myopic fundus changes of chorioretinal degeneration $(\mathrm{M})$ were graded using the classification published by Avila et al. ${ }^{22}$ The staphyloma of all the patients was classified according to the classification published by Steidl and Pruett. ${ }^{23}$ Briefly, the staphyloma were graded from zero to four based on the depth of the staphyloma measured on B-scan ultrasonographic images. An elongated eye with a smooth scleral contour was graded as zero. A grade 1 staphyloma had a depth of $2 \mathrm{~mm}$ or less, a grade 2 staphyloma had a depth of $>2 \mathrm{~mm}$ but $<4 \mathrm{~mm}$, a grade 3 staphyloma had a depth of $>4 \mathrm{~mm}$ but $<6 \mathrm{~mm}$ and a grade 4 staphyloma had a depth of $>6 \mathrm{~mm}$. The vitreous was carefully examined in each patient for a posterior vitreous detachment or remaining vitreous attachments to the retina with binocular indirect ophthalmoscope, SD-optical coherence tomography and visualisation of the vitreous with triamcinolone acetonide intraoperatively. All cases were followed-up at 1, 3, 6, 9, 12, 18 and 24 months postoperatively. The primary outcome measure was defined as initial retinal reattachment rate. Secondary outcomes included macular holes closure rate, and the changes of BCVA (LogMAR), axial lengths and IOP. If retinal detachment recurred after the surgery, the patient was offered silicone oil endotamponade for repair within 2 weeks of presentation.

\section{Statistical analysis}

Basal homogeneity between the two groups was studied using the $\chi^{2}$ test, Fisher's exact test or the Mann-Whitney $U$ test for qualitative or quantitative variables, respectively. Between the two groups, statistical comparison of mean BCVA (LogMAR), axial length and IOP before and after surgery was performed using Wilcoxon matched pairs signed rank test, and the anatomical success was compared with $\chi^{2}$ test or Fisher's exact test. All statistical analyses were performed using SPSS V.20.0 software, and $\mathrm{p}<0.05$ was used for statistical significance.

\section{RESULTS}

One hundred thirty-one eyes of 131 patients with MHRD initially were assessed for eligibility. Of these patients, 107 patients were recruited in this study, who wrote the informed consent and were randomly assigned to the two groups. Nine patients could not complete this study as planned for several kinds of reasons including deceased of unrelated causes $(n=1)$, physically unable to return for the follow-up $(n=4)$ and loss of contact $(n=4)$. At the end of the 24-month follow-up, 98 eyes (91.6\%) enrolled patients completed the study; 52 eyes in group 1 and 46 eyes in group 2 were included in the analysis. Demographic and preoperative ophthalmic data of the two groups were described in table 1 and no statistically significant differences were observed regarding any of these variables $(p>0.05)$. The grades of chorioretinal degeneration of the two groups were M4 or M5 and all eyes had grade 4 staphyloma (table 1). There were no intraoperative or postoperative complications such as intraocular haemorrhage, choroidal detachment and proliferative vitreoretinopathy occurred in all cases of the two groups.

\section{Anatomical outcomes}

Retinas were reattached in all cases of two groups 1 month postoperatively. The initial retinal reattachment rate was $96.15 \%$ (50 eyes), $88.46 \%$ (46 eyes), 82.69\% (43 eyes), $76.92 \%$ (40 eyes), 75\% (39 eyes) and 75\% (39 eyes) in group 1 , and 100\% (46 eyes), 97.83\% (45 eyes), 93.48\% (43 eyes), 
Table 1 Demographic and preoperative ophthalmic data of the study participants

\begin{tabular}{|c|c|c|c|}
\hline Characteristics & Group 1 & Group 2 & p Value \\
\hline No. of patients (eyes) & $52(52)$ & $46(46)$ & \\
\hline Gender (female/male) & $24 / 28$ & $22 / 24$ & $0.869^{*}$ \\
\hline Age (years) & $48.70(10.21)$ & $53.11(13.52)$ & $0.31 \dagger$ \\
\hline \multicolumn{4}{|l|}{ Refraction (dioptres) } \\
\hline Mean (SD) & $-21.20(4.05)$ & $-23.62(4.72)$ & $0.32 \dagger$ \\
\hline Median (p25; p75) & $21.50(-19.50 ;-25.50)$ & $-23(-20 ;-25)$ & \\
\hline ( $\min$ to $\max )$ & $(-17$ to -30$)$ & $(-18$ to -29$)$ & \\
\hline Axial length (mm) & & & $0.270 \dagger$ \\
\hline Mean (SD) & $32.73(2.42)$ & $33.98(2.35)$ & \\
\hline Median (p25; p75) & $33.53(31.00 ; 34.00)$ & 33.05 (31.50; 34.05) & \\
\hline ( $\min$ to $\max )$ & 30.10 to 36.00 & 30.00 to 37.50 & \\
\hline \multicolumn{4}{|l|}{ BCVA (LogMAR) } \\
\hline Mean (SD) & $1.67(0.49)$ & $1.61(0.53)$ & $0.404 \dagger$ \\
\hline Median (p25; p75) & $1.602(1.00 ; 1.903)$ & $1.602(0.80 ; 1.903)$ & \\
\hline ( $\min$ to $\max )$ & 0.70 to 2.30 & 0.50 to 2.30 & \\
\hline Retinoschisis-like feature, $\mathrm{n}(\%)$ & $25(48.10 \%)$ & $26(56.50 \%)$ & $0.403^{*}$ \\
\hline Lens status, n (\%) & & & $0.194^{*}$ \\
\hline Aphakic & 0 & 0 & \\
\hline Phakic & $26(50 \%)$ & $29(63 \%)$ & \\
\hline Pseudophakic & $26(50 \%)$ & $17(37 \%)$ & \\
\hline \multicolumn{4}{|l|}{ IOP $(\mathrm{mm} \mathrm{Hg})$} \\
\hline Mean (SD) & $14.60(3.21)$ & $15.12(3.30)$ & $0.306 t$ \\
\hline Median (p25; p75) & $14.9(13.8 ; 16.3)$ & $15.2(13.5 ; 16.1)$ & \\
\hline ( $\min$ to $\max )$ & 12.1 to 17.5 & 13.0 to 17.5 & \\
\hline \multicolumn{4}{|l|}{ Symptom duration (months) } \\
\hline Mean (SD) & $4.78 \pm 3.32$ & $6.10 \pm 4.21$ & $0.672 \dagger$ \\
\hline Median (p25; p75) & $4.5(2.5 ; 6.0)$ & $5.5(3.5 ; 7.0)$ & \\
\hline (min to $\max )$ & 0.5 to 10.5 & 1 to 12 & \\
\hline PVD presence, $\mathrm{n}(\%)$ & $11(21.15 \%)$ & $7(15.22 \%)$ & $0.449^{*}$ \\
\hline \multicolumn{4}{|l|}{ PVR (posterior) } \\
\hline Grade B & $44(88.46 \%)$ & $40(91.30 \%)$ & $0.741^{*}$ \\
\hline Grade C & $8(11.54 \%)$ & $6(8.70 \%)$ & \\
\hline Grades of chorioretinal degeneration§, $n(\%)$ & & & $0.609^{*}$ \\
\hline Grade M4 & $23(44.23 \%)$ & $18(39.13 \%)$ & \\
\hline Grade M5 & $29(55.77 \%)$ & $28(60.87 \%)$ & \\
\hline \multicolumn{4}{|l|}{ Grades of posterior staphylomaף, $\mathrm{n}(\%)$} \\
\hline Grade 4 & $52(100 \%)$ & $46(100 \%)$ & \\
\hline Area of retinal detachment & & & $0.418^{*}$ \\
\hline Within staphyloma & $24(46.15 \%)$ & $25(54.35 \%)$ & \\
\hline Beyond staphyloma & $28(53.85)$ & $21(45.65 \%)$ & \\
\hline
\end{tabular}

93.48\% (43 eyes), 89.13\% (41 eyes) and 89.13\% (41 eyes) in group 2 , at $3,6,9,12,18$ and 24 months postoperatively, respectively (figure $3 \mathrm{~A}$ ). The rate of initial retinal reattachment was significantly higher in group 2 than in group 1 at 12 -month postoperatively $\left(\chi^{2}\right.$ test, $\left.\mathrm{p}=0.020\right)$. Until the end of follow-up, retinal detachment recurred in 13 eyes $(13 / 52,25 \%)$ in group 1 and 5 eyes $(5 / 46,10.87 \%)$ in group 2 , which were caused totally by macular hole. All cases were reattached after a second surgery of silicone oil endotamponade, and all silicone oil were removed successfully 3-4 months postoperatively in reoperative cases. Until the end of follow-up, the macular hole closed (Figure $1 \mathrm{H})$ in 1 eye $(1 / 46,2.17 \%), 5$ eyes $(5 / 46$,
$10.87 \%), 8$ eyes $(8 / 46,17.39 \%), 9$ eyes $(9 / 46,19.57 \%), 12$ eyes $(12 / 46,26.09 \%), 12$ eyes $(12 / 46,26.09 \%)$ and 12 eyes $(12 / 46,26.09 \%)$ in group 2 , and 0 eye $(0 / 52,0 \%), 0$ eye $(0 / 52$, $0 \%), 3$ eyes (3/52, $5.77 \%), 4$ eyes $(4 / 52,7.69 \%), 4$ eyes $(4 / 52$, $7.69 \%), 3$ eyes $(3 / 52,5.77 \%)$ and 2 eyes $(2 / 52,3.85 \%)$ in group 1 at $1,3,6,9,12,18$ and 24 months postoperatively, respectively (figure $3 \mathrm{~B}$ ). The macular hole close rate was significantly different between the two groups at 3, 12, 18 and 24 months postoperatively (Fisher's exact test, $\mathrm{p}<0.05$ ). The reopening of previously closed macular hole was not found in group 2, but occurred in two cases in group 1 at 18, 24 months postoperatively, respectively (figure $3 \mathrm{~B}$ ). 
Figure 3 Anatomical outcome comparison between two groups. The difference between the two groups were significant in the initial retinal reattachment $(\mathrm{A})$ at 12 months postoperatively ( ${ }^{*} p<0.05$, Fisher's exact test), and in macular hole closure rate $(B)$ at $3,12,18$ and 24 months postoperatively ( ${ }^{*} p<0.05, \chi^{2}$ test).
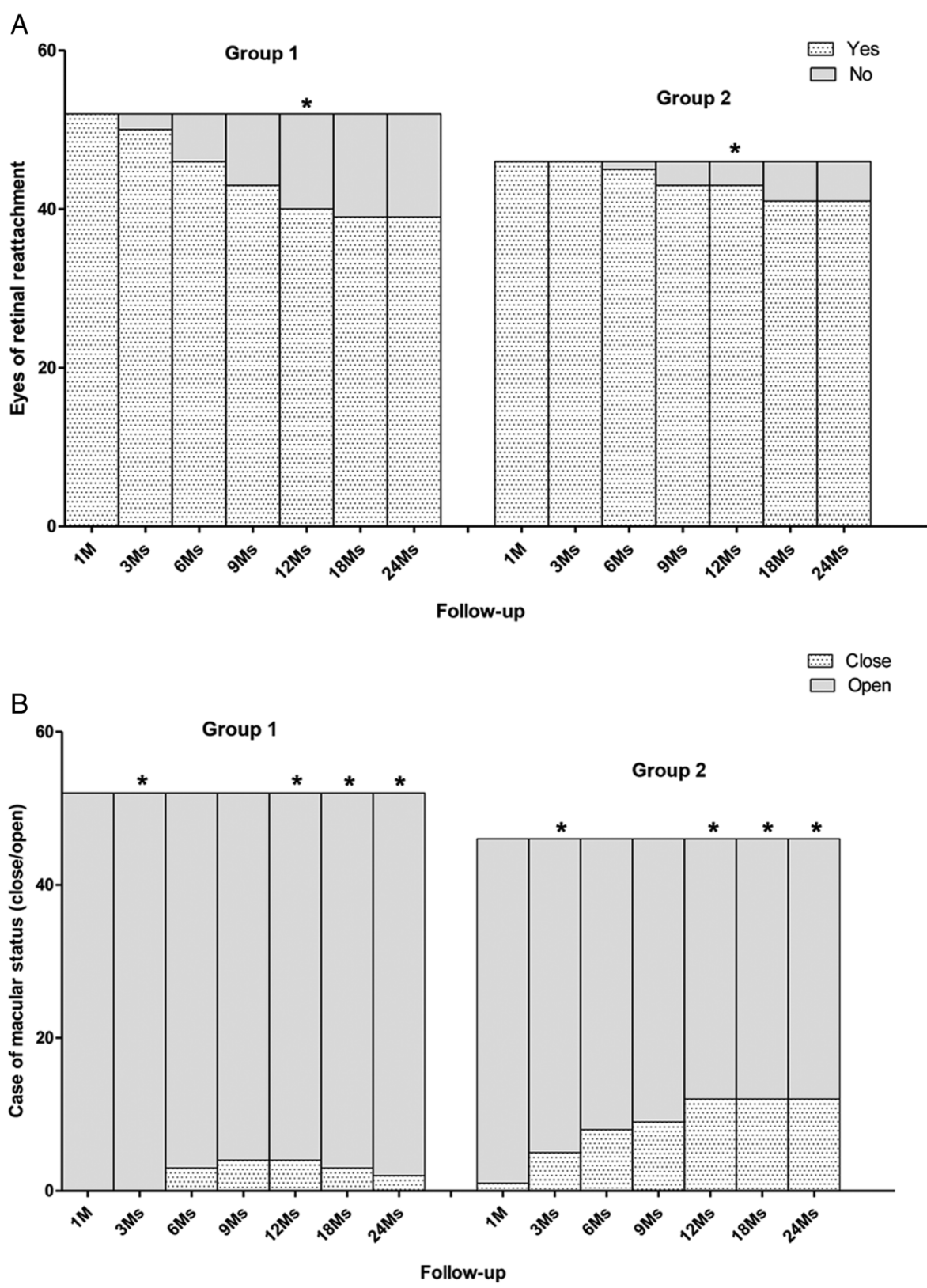

\section{Best-corrected visual acuity}

There was no significant difference between the two groups in preoperative BCVA (LogMAR) (Mann-Whitney U test, $\mathrm{p}=0.404)$ (table 1). The mean BCVA (LogMAR) changes of initial retinal reattached eyes in group 2 was worse than in group 1 at 1 and 3 months postoperatively, and the difference was significant at 1 month postoperatively (table 2) (figure 4A) (Wilcoxon matched pairs signed rank test, $\mathrm{p}=0.036$ ). Since 6 months postoperatively, the mean BCVA (LogMAR) improvement of initial retinal reattached eyes were significantly greater in group 2 than in group 1 at each visit point (Wilcoxon matched pairs signed rank test, $\mathrm{p}<0.05$ ) (table 2) (figure 4A). In initial retinal reattachment cases of group 2, the postoperative BCVA was significantly better in patients with macular hole closure than in those with persistent holes (Mann-Whitney $\mathrm{U}$ test, $\mathrm{p}<0.05)$. At the end of follow-up, the mean BCVA (LogMAR) of retinal redetachment eyes before a second surgery was significantly better in group $2(1.36 \pm 0.13)$ than in group 1 $(2.02 \pm 0.41)$ (Wilcoxon matched pairs signed rank test, $\mathrm{p}<0.05$ ) (table 2).

\section{Axial lengths}

The convexity of posterior eye wall including posterior staphyloma was trended to be flattened after macular buckle until the final visit (figure 1I). Preoperative and postoperative axial length at $1,3,6,9,12,18$ and 24 months postoperatively were 32.73 $\pm 2.42,32.91 \pm 3.29,32.80 \pm 2.95,32.70 \pm 2.06,32.78 \pm 2.33$, $32.76 \pm 2.17,32.85 \pm 3.01$ and $32.90 \pm 3.20 \mathrm{~mm}$, respectively in group 1 , and were $33.98 \pm 2.35,28.09 \pm 2.77,27.73 \pm 2.26$, $29.11 \pm 3.15,29.20 \pm 2.17,29.09 \pm 3.08,29.26 \pm 2.94$ and 30.02 $\pm 2.51 \mathrm{~mm}$, respectively in group 2 (figure $4 \mathrm{~B}$ ). The mean axial length in group 2 had shortened more significantly than in group 1 at each follow-up time point (Wilcoxon matched pairs signed rank test, $\mathrm{p}<0.05$ ). In group 2 , the mean postoperative axial length was reduced by $4.66 \pm 1.77 \mathrm{~mm}$ until the end of visit.

\section{Intraocular pressure}

Preoperative and postoperative IOP at 1, 3, 6, 9, 12, 18 and 24 months postoperatively were $14.46 \pm 3.21,22.22 \pm 4.07$, $19.50 \pm 4.00,16.53 \pm 3.66,18.15 \pm 3.35, \quad 17.30 \pm 3.78,15.34$ 
Table 2 Changes in preoperative and postoperative BCVA and retina status of two groups

\begin{tabular}{|c|c|c|c|c|c|c|}
\hline \multirow{2}{*}{$\begin{array}{l}\text { Preoperation and } \\
\text { follow-up (months) }\end{array}$} & \multicolumn{2}{|c|}{$\begin{array}{l}\text { Group } 1 \text { (no. of eyes, LogMAR } \\
\text { BCVA (mean } \pm \text { SD)) }\end{array}$} & \multicolumn{2}{|c|}{$\begin{array}{l}\text { Group } 2 \text { (no. of eyes, LogMAR } \\
\text { BCVA (mean } \pm \text { SD)) }\end{array}$} & \multirow{2}{*}{$\begin{array}{l}\text { p Value of initial } \\
\text { success cases } \\
\text { (group } 1 \text { vs group 2) }\end{array}$} & \multirow{2}{*}{$\begin{array}{l}\text { p Valuet of BCVA in } \\
\text { initial success } \\
\text { (group } 1 \text { vs group } 2 \text { ) }\end{array}$} \\
\hline & Initial success & Initial failure & Initial success & Initial failure & & \\
\hline Preoperation & $52,(1.67 \pm 0.49)$ & & $46(1.61 \pm 0.53)$ & & & \\
\hline 1 & $52(1.31 \pm 0.45)$ & 0 & $46(1.46 \pm 0.43)$ & 0 & & $0.036^{*}$ \\
\hline 3 & $50(1.26 \pm 0.31)$ & $2(2.10 \pm 0.28)$ & $46(1.35 \pm 0.37)$ & 0 & $0.502 \ddagger$ & 0.080 \\
\hline 6 & $46(1.13 \pm 0.33)$ & $6(1.95 \pm 0.39)$ & $45(0.93 \pm 0.30)$ & $1(1.60)$ & $0.161 \S$ & $0.012^{*}$ \\
\hline 9 & $43(1.11 \pm 0.37)$ & $9(1.95 \pm 0.39)$ & $43(0.90 \pm 0.28)$ & $3(1.40 \pm 0.17)$ & $0.103 \S$ & $0.010^{*}$ \\
\hline 12 & $40(1.13 \pm 0.28)$ & $12(2.11 \pm 0.45)$ & $43(0.90 \pm 0.32)$ & $3(1.40 \pm 0.17)$ & $0.022 * \S$ & $0.007^{*}$ \\
\hline 18 & $39(1.22 \pm 0.35)$ & $13(2.02 \pm 0.41)$ & $41(0.98 \pm 0.29)$ & $5(1.36 \pm 0.13)$ & $0.070 \S$ & $0.007^{*}$ \\
\hline 24 & $39(1.30 \pm 0.43)$ & $13(2.02 \pm 0.41)$ & $41(1.05 \pm 0.40)$ & $5(1.36 \pm 0.13)$ & $0.070 \S$ & $0.007^{*}$ \\
\hline
\end{tabular}

Figure 4 Preoperative and postoperative evaluation of best-corrected visual acuity (BCVA) (LogMAR) (A), axial length (B) and intraocular pressure (IOP) (C) (Wilcoxon matched pairs signed rank test). The different change between the two groups were significant in BCVA (LogMAR) of initial retinal reattached cases at 1, 6, 9, 12, 18, 24 months postoperatively $(p<0.05)$, in axial length of all eyes at each follow-up point $(p<0.05)$ and in IOP of all eyes at 1 month postoperatively $(p=0.041)$.

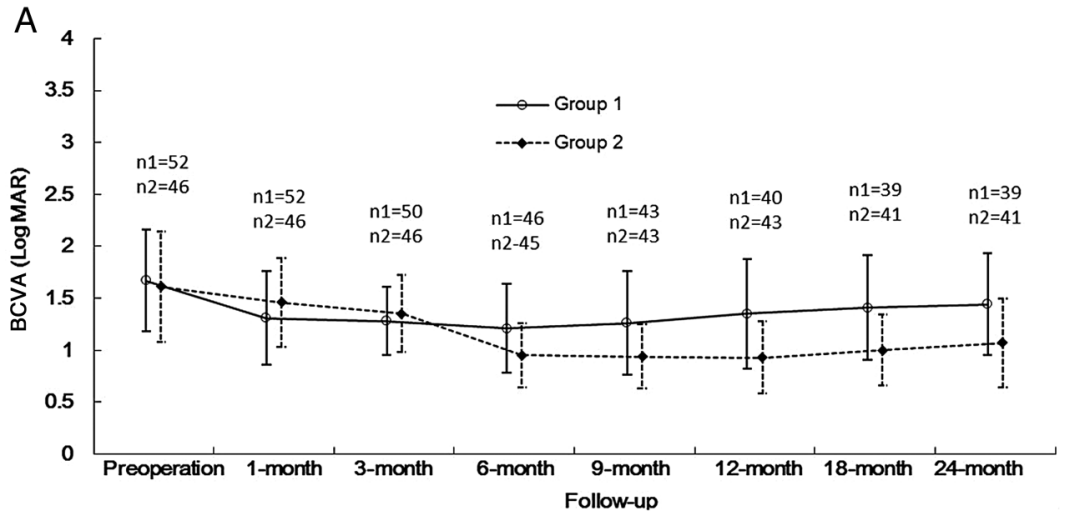

B

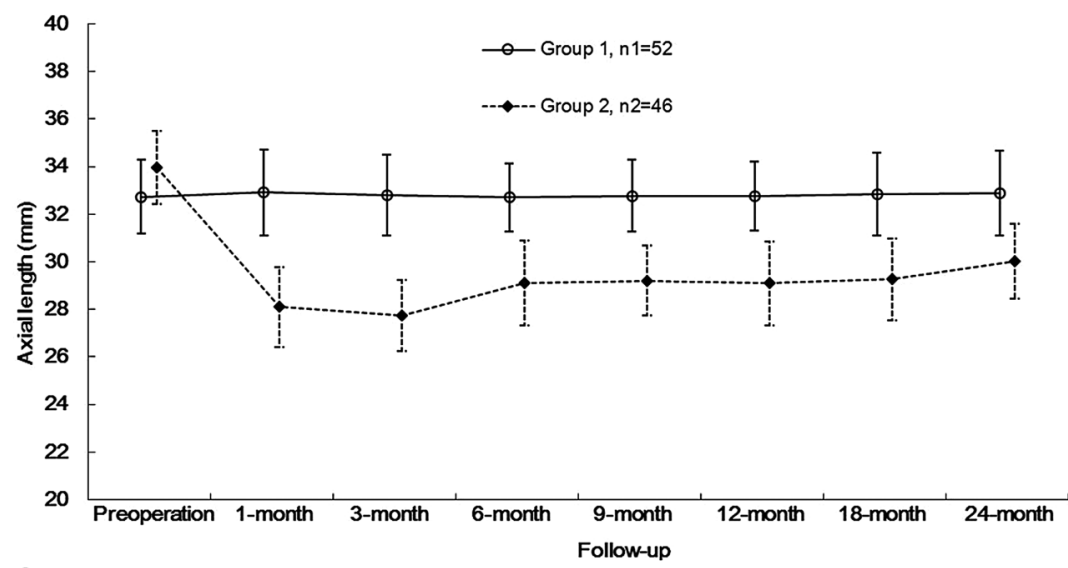

C

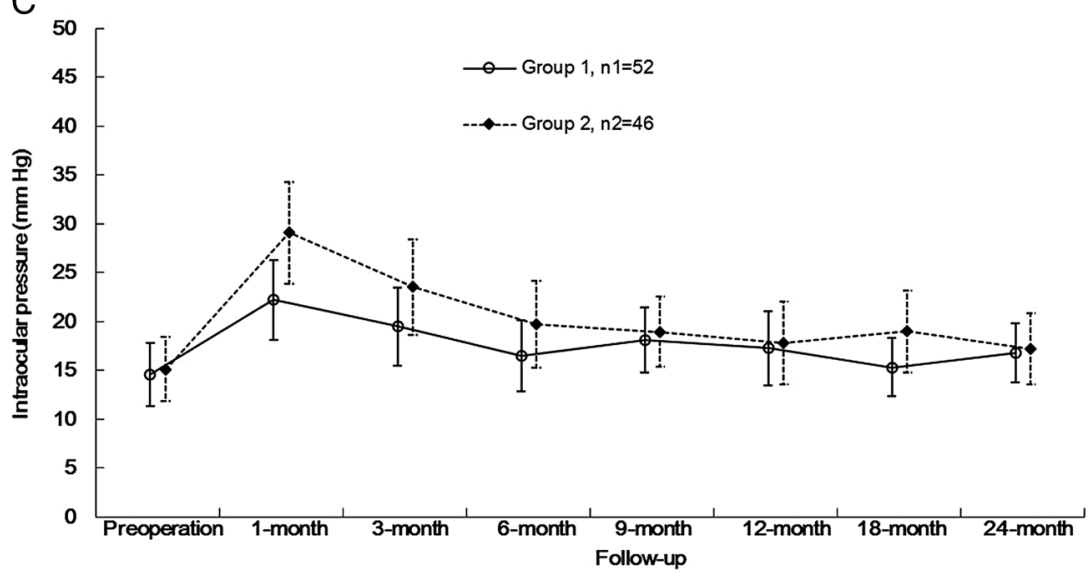


\pm 2.97 and $16.78 \pm 3.02(\mathrm{~mm} \mathrm{Hg})$, respectively in group 1 , and $15.12 \pm 3.30,29.10 \pm 5.18,23.55 \pm 4.92,19.70 \pm 4.44,18.95$ $\pm 3.60,17.80 \pm 4.21,19.00 \pm 4.22$ and $17.21 \pm 3.59(\mathrm{~mm} \mathrm{Hg})$, respectively in group 2 (figure $4 \mathrm{C}$ ). The IOP was increased significantly 1 month postoperatively in group 2 than in group 1 (Wilcoxon matched pairs signed rank test, $\mathrm{p}=0.041$ ). There was no significant difference in IOP change between the two groups since 3 months postoperatively (Wilcoxon matched pairs signed rank test, $\mathrm{p}>0.05$ ). No patient developed durative glaucoma and underwent filtration surgery.

\section{DISCUSSION}

High myopia and longer axial length are known to be related to worse prognosis of retinal detachment and macular hole, ${ }^{24-27}$ especially for patients with axial length $>30 \mathrm{~mm} \cdot{ }^{28} 29$ In patients with MHRD with extreme high axial myopia, the recurrence rate is very high. This is because traditional PPV could not address all of the pathogenic factors involved in MHRD with extreme high axial myopia. Improvement of surgical techniques including posterior hyaloid detachment, ILM peeling and the use of silicone oil could not prevent recurrence of retinal detachment and reopening macular hole. ${ }^{30}$ Some patients even require multiple surgeries to achieve retinal reattachment due to the loss of chorioretinal tissue and retinal pigment epithelial atrophy. This is the reason for the revival of macular buckling to deal with these cases. ${ }^{30}$ However, macular buckle has always been difficult and hazardous, particularly in the highly myopic eyes. This is largely caused by difficulty in localising the macular hole on the episclera and the lack of understanding of the relationships of important structures at the posterior region of the globe. ${ }^{30}$ In this study, we have designed a new surgical technique of macular buckling under direct vision in combination with PPV and ILM peeling in the treatment of MHRD in patients with high axial myopia $(>30 \mathrm{~mm}$ ), and conducted a prospective comparative study with a longterm follow-up of 24 months.

In this study, the primary outcome measures were initial retinal reattachment. The combined buckle/PPV surgery was effective in achieving retinal reattachment at 12 months postoperatively. However, after a longer follow-up of $>12$ months, the effect was not significant again. Therefore, further clinical studies for a longer-term follow-up should be considered to assess the true long-term efficiency on retinal reattachment of this technique. Also, the macular buckling and PPV with ILM peeling surgery could even facilitate the macular hole closure since 3 months postoperatively, and there was no macular hole reopen case occurred after surgery. It might be explained by a greater tightly contact surface area between the retina and atrophied choroid/exposed bare sclera at the macular hole region due to the buckling crest at the staphyloma, thus facilitating glial cell proliferation and macular hole closure.

More promisingly, combined buckle/PPV surgery has also achieved greater BCVA improvement since 6 months postoperatively. The BCVA increase was in accordance with the rate of macular hole closure, which was similar to the previous studies that postoperative BCVA were significantly better in patients with macular hole closure than in those with persistent holes. $^{31} 32$ However, it must be noted that at approximately 1 month postoperatively, BCVA of patients that had undergone combined buckle/PPV surgery showed a temporary decrease instead. This might be due to the surgical intervention of episcleral buckle affecting the choroidal and retinal circulation, causing transient postoperative inflammation at the posterior staphyloma, and restricting the BCVA improvement. In addition, in those cases of recurrent retinal detachment, the BCVA reduction was significantly less after the combined buckle/PPV operation than that after the PPV operation alone. The reason might be the reshaping of the posterior scleral wall after macular indentation, which reshaped the deep posterior staphyloma from extremely concave into a flat configuration. Reccurent retinal detachment should be generally more extensive and severe after PPV surgery, because the vitreous is filled with fluid instead of primary vitreous gel and the fluid flows more freely into the subretinal space from the vitreous cavity through macular hole, thus causing a greater extent of retinal detachment at the posterior region. However, to a certain extent, under the condition of recurrent retinal detachment with macular indentation, the distance between posterior pole of retina and scleral wall could be shortened, which reduced fluid flowing into subretinal space and lessens the height of retinal redetachment.

The axial length shortening and IOP elevation were commonly accompanied by the result of macular buckle, and correlated with the height and tension of episcleral buckle. ${ }^{33}{ }^{34}$ In this study, the postoperative axial lengths have kept shortening significantly after combined buckle/PPV surgery until the final visit. Thus, the new designed macular buckling is helping to maintain the buckle height in the long term, and the buckling element of silicone tire could be stable and effective for a longterm extrascleral compression. However, the surgical efficiency of shortening axial length was gradually decreased during follow-up period (figure 4B). It is the reason that silicone band has an inherent elasticity, and the decreasing elasticity of the silicone band with time could cause a weakening of pressure exerted on the sclera. Furthermore, the stretching of stressed scleral tissue after buckle intervention might also contribute to the regain of axial length. Thus, we need a more expansive clinical study with long-term follow-up to assess the true nature of long-term stability in combined buckle/PPV surgery. Besides, the measurement of postoperative axial length was an extremely valuable indicator of therapeutic efficiency in macular buckle. ${ }^{15}$ The presence of a posterior staphyloma could lead to significant errors in A-scan ultrasonography axial length measurements because the anatomic axial length (the distance from the corneal vertex to the posterior pole) might differ from the refractive axial length (the distance from the corneal vertex to the fovea), ${ }^{35}$ or in B-scan ultrasonography axial length measurements for inability to consistently locate the centre of the fovea. ${ }^{36}$ We had overcome this challenge by using optical coherence biometry (IOL Master) in this study, avoiding the challenges in measuring eyes with posterior staphyloma and extending the axial length measurement to the level of the retinal pigment epithelium to avoid retinal pathologies such as retinal detachment. ${ }^{12}$ Furthermore, IOP elevation was also a common complication of macular buckling and a cause of concern. $^{34}$ The mechanical compression due to indentation caused by the macular buckle might be the major reason for IOP elevation, especially in eyes with extremely high axial length that had thinner eye wall and greater exposure of choroid vessels. In this study, we had observed a transient increase in IOP 1 month after combined buckle/PPV surgery, which was mostly self-limited and had reduced to normal levels since 3 months postoperatively. This temporary increase in IOP might be caused by postoperative swelling and inflammation of the eyeball soft tissue. As time goes on postoperatively, with the gradually relieved swelling and resolved inflammation, the power of scleral indentation was loosened a little, and the postoperative short-term increased IOP resumed normal. Therefore, 
this study designed a combined buckle/PPV surgery, which could reduce the adverse effect of severe postoperative IOP elevation as it is performed under direct vision and more accurate adjustments can be made to control cerclage tension and indentation strength, although it could accompanied with short-term and self-limited postoperative IOP increasing. Tortuous or congested vortex vein was intraoperative indicators of overcompression on the eyeball which could cause postoperative IOP elevation and should be avoided during surgery (figure1C).

It is reported that macular buckling could effectively reduce axial length of the eye and correct posterior staphyloma by changing the concave configuration of the posterior pole into a convex one. ${ }^{37}$ In this study, however, the shortening axial length was not a large amount, because the indentation of the buckle induced a more flattening of the posterior staphyloma and not an inversion of the shape of the staphyloma. The postoperative mean axial length was only reduced by $4.66 \pm 1.77 \mathrm{~mm}$ during the whole follow-up period. Thus, the strength of macular buckling indentation in this study was relatively moderate. Recent studies have revealed that ${ }^{11} 3738$ macular buckling alone could be an effective method for treating MHRD in high myopic eyes. But in the case of extreme high axial length, only a much deeper macular indentation with more strong compression on the posterior staphyloma could be effective in MHRD treatment by macular buckling alone surgery, which could induce much more postoperative complications such as severe IOP elevation or even eyewall ischaemia. ${ }^{15}$ In this study, we designed a combined surgery of PPV with ILM peeling and macular buckle to treat the MHRD with extreme high axial length, which acquired interior compression (gas tamponade) and exterior indentation (macular buckle) on macular hole area. PPV with ILM peeling could remove the tangential and centripetal tractions caused by vitreous cortex, epiretinal membranes or ILM, ${ }^{10} 20$ and macular buckle could correct the disproportion between the retina and the elongated sclera and relieved the centripetal tractions. ${ }^{14} 39$ The combined procedures in this study achieved a better retinal reattachment and high macular hole closure rate, and also avoid the excessive macular indentation associated with many adverse effects.

In comparison to other traditional buckling methods, the new designed combined buckle/PPV surgery in this study is more convenient and yield better results. Many different buckling techniques have been proposed in the history of the surgery of high myopia, including a radially placed polyethylene tube, ${ }^{40} \mathrm{a}$ silver ring, later modified, attached to the limbus with an arm fixed to the ring with a terminal ball to indent the retina, ${ }^{41}{ }^{42}$ a vertically placed $2 \mathrm{~mm}$ thick silastic $\operatorname{rod}^{43}$ and an oblique circlage, ${ }^{44}$ a silastic sponge rod placed between the inferior oblique insertion and the optic nerve. ${ }^{6}$ Although some surgical performance is effective due to accurate identification of constant landmarks of the fovea and long posterior ciliary arteries in relation to inferior oblique insertion, ${ }^{30}$ this is extremely difficulty in highly myopic eyes due to this relative locality can be varied because of the prolonged axial length and the extension of the posterior segment. Therefore, it is difficult to localise the mattress exactly under the macular, neither adjust the buckle to produce effective macular indentation. All the listed buckle designs have been abandoned for the inability to check the exact position of the buckle intraoperatively, and for intraoperative or postoperative complications and the surgical challenge. ${ }^{45}$ Exact localisation and adjustment of the buckle to produce effective macular indentation is a challenge. Although new techniques including scleral imbrication combined with PPV, ${ }^{12}$ posterior scleral reinforcement ${ }^{46}$ and L-shaped buckle made of silicone sponge and titanium stent ${ }^{15}$ have been raised, these methods are inadequate in their extent of macular indentation and their buckling efficacy were uncertain.

The major advantage in this study depended on the fact that buckle could be performed under direct vision and possessed the ability to pinpoint the exact position of macular indentation. The intraoperative adjustments could be made more accurately to determine the strength of circlage and the height of the macular buckle, especially in cases of highly myopic MHRD with axial length $>30 \mathrm{~mm}$. Furthermore, this technique need not be a complex posterior episcleral suture, and can be performed without needing specially designed buckling elements, which can be made available anywhere. Certainly, as a small-scale study, we still require more expansive clinical studies that are necessary in order to give a more precise evaluation of validity in this procedure, especially in retinal reattachment or the stability of macular indentation.

Contributors LL conceived and designed the study; JM was operator in this study, and drafted this article; HL, XD and ST collected, analysed and interpreted the clinical data and revised the manuscript.

Funding This work was supported by the National Natural Science Foundation of Guangdong, China (grant number 2016A030313364) and the Science and Technology Program of Guangzhou, China (grant number 2016070010070).

Competing interests None declared.

Patient consent Obtained.

Ethics approval The institutional ethics committee of Zhongshan Ophthalmic Center, Sun Yat-Sen University.

Provenance and peer review Not commissioned; externally peer reviewed.

Open Access This is an Open Access article distributed in accordance with the Creative Commons Attribution Non Commercial (CC BY-NC 4.0) license, which permits others to distribute, remix, adapt, build upon this work non-commercially, and license their derivative works on different terms, provided the original work is properly cited and the use is non-commercial. See: http://creativecommons.org/ licenses/by-nc/4.0/

\section{REFERENCES}

1 Akiba J, Konno S, Yoshida A. Retinal detachment associated with a macular hole in severely myopic eyes. Am J Ophthalmol 1999;128:654-5.

2 Siam A. Macular hole with central retinal detachment in high myopia with posterior staphyloma. Br J Ophthalmol 1969;53:62-3.

3 Alkabes $M$, Burés-Jelstrup A, Salinas C, et al. Macular buckling for previously untreated and recurrent retinal detachment due to high myopic macular hole: a 12-month comparative study. Graefes Arch Clin Exp Ophthalmol 2014;252:571-81.

4 Morita $\mathrm{H}$, Ideta $\mathrm{H}$, Ito $\mathrm{K}$, et al. Causative factors of retinal detachment in macular holes. Retina 1991;11:281-4.

5 Aaberg TM, Blair CJ, Gass JD. Macular holes. Am J Ophthalmol 1970;69:555-62.

6 Theodossiadis GP, Sasoh M. Macular buckling for retinal detachment due to macular hole in highly myopic eyes with posterior staphyloma. Retina 2002;22:129.

7 Ando F, Ohba N, Touura K, et al. Anatomical and visual outcomes after episcleral macular buckling compared with those after pars plana vitrectomy for retinal detachment caused by macular hole in highly myopic eyes. Retina 2007;27:37-44.

8 Bures-Jelstrup A, Alkabes M, Gomez-Resa M, et al. Visual and anatomical outcome after macular buckling for macular hole with associated foveoschisis in highly myopic eyes. Br J Ophthalmol 2013;98:104-9.

9 Nadal J, Verdaguer P, Canut MI. treatment of retinal detachment secondary to macular hole in high myopia: vitrectomy with dissection of the inner limiting membrane to the edge of the staphyloma and long-term tamponade. Retina 2012;32:1525-30.

10 Nakanishi H, Kuriyama S, Saito I, et al. Prognostic factor analysis in pars plana vitrectomy for retinal detachment attributable to macular hole in high myopia: a multicenter study. Am J Ophthalmol 2008;146:198-204.

11 Ripandelli G, Coppe AM, Fedeli R, et al. Evaluation of primary surgical procedures for retinal detachment with macular hole in highly myopic eyes: a comparison [corrected] of vitrectomy versus posterior episcleral buckling surgery. Ophthalmology 2001;108:2258-64; discussion2265.

12 Fujikawa M, Kawamura H, Kakinoki M, et al. Scleral imbrication combined with vitrectomy and gas tamponade for refractory macular hole retinal detachment associated with high myopia. Retina 2014;34:2451-7.

13 Stirpe M, Ripandelli G, Rossi T, et al. A new adjustable macular buckle designed for highly myopic eyes. Retina 2012;32:1424-7. 
14 Baba T, Tanaka S, Maesawa A, et al. Scleral buckling with macular plombe for eyes with myopic macular retinoschisis and retinal detachment without macular hole. Am J Ophthalmol 2006;142:483-7.

15 Parolini B, Frisina R, Pinackatt S, et al. Indications and results of a new I-shaped macular buckle to support a posterior staphyloma in high myopia. Retina 2015:35:2469-82.

16 Mura M, lannetta D, Buschini E, et al. T-shaped macular buckling combined with $25 \mathrm{G}$ pars plana vitrectomy for macular hole, macular schisis, and macular detachment in highly myopic eyes. Br J Ophthalmol 2017;101:383-8.

17 Kuriyama S, Matsumura M, Harada T, et al. Surgical techniques and reattachment rates in retinal detachment due to macular hole. Arch Ophthalmol 1990;108:1559-61.

18 Mancino R, Ciuffoletti E, Martucci A, et al. Anatomical and functional results of macular Hole retinal detachment surgery in patients with high myopia and posterior staphyloma treated with perfluoropropane gas or silicone oil. Retina 2013;33:586-92

19 Suda K, Hangai M, Yoshimura N. Axial length and outcomes of macular hole surgery assessed by spectral-domain optical coherence tomography. Am J Ophthalmol 2011;151:118-27.

20 Nishimura A, Kimura $M$, Saito $Y$, et al. Efficacy of primary silicone oil tamponade for the treatment of retinal detachment caused by macular hole in high myopia. Am J Ophthalmol 2011;151:148-55.

21 Krzystolik K, Stopa M, Kuprjanowicz L, et al. Pars Plana vitrectomy in advanced cases of von Hippel-Lindau eye disease. Retina 2016;36:325-34.

22 Avila MP, Weiter JJ, Jalkh AE, et al. Natural history of choroidal neovascularization in degenerative myopia. Ophthalmology 1984:91:1573-81.

23 Steidl SM, Pruett RC. Macular complications associated with posterior staphyloma. Am J Ophthalmol 1997:123:181-7.

24 Kobayashi H, Kobayashi K, Okinami S. Macular hole and myopic refraction. Br J Ophthalmol 2002;86:1269-73.

25 Patel SC, Loo RH, Thompson JT, et al. Macular hole surgery in high myopia. Ophthalmology 2001;108:377-80.

26 García-Arumí J, Martinez V, Puig J, et al. The role of vitreoretinal surgery in the management of myopic macular hole without retinal detachment. Retina 2001;21:332-8.

27 Sulkes DJ, Smiddy WE, Flynn HW, et al. Outcomes of macular hole surgery in severely myopic eyes: a case-control study. Am J Ophthalmol 2000;130:335-9.

28 Olenik A, Rios J, Mateo C. Inverted internal limiting membrane flap technique for macular holes in high myopia with axial length $\geq 30 \mathrm{~mm}$. Retina 2016;36: 1688-93.

29 Alkabes M, Pichi F, Nucci P, et al. Anatomical and visual outcomes in high myopic macular hole (HM-MH) without retinal detachment: a review. Graefes Arch Clin Exp Ophthalmol 2014;252:191-9.
30 Siam AL, El Maamoun TA, Ali MH. Macular buckling for myopic macular hole retinal detachment: a new approach. Retina 2012:32:748-53.

31 Ikuno Y, Sayanagi K, Oshima T, et al. Optical coherence tomographic findings of macular holes and retinal detachment after vitrectomy in highly myopic Eyes. Am J Ophthalmol 2003;136:477-81.

32 Lam RF, Lai WW, Cheung BT, et al. Pars Plana vitrectomy and perfluoropropane (C3F8) tamponade for retinal detachment due to myopic macular hole: a prognostic factor analysis. Am J Ophthalmol 2006;142:938-44.

33 Phelps CD, Burton TC. Glaucoma and retinal detachment. Arch Ophthalmol 1977:95:418-22

34 Perez RN, Phelps CD, Burton TC. Angel-closure glaucoma following scleral buckling operations. Trans Sect Ophthalmol Am Acad Ophthalmol Otolaryngol 1976:81:247-52.

35 Hsiang HW, Ohno-Matsui K, Shimada N, et al. Clinical characteristics of posterior staphyloma in eyes with pathologic myopia. Am J Ophthalmol 2008;146:102-10.

36 Shen $P$, Zheng $Y$, Ding $X$, et al. Biometric measurements in highly myopic eyes. I Cataract Refract Surg 2013:39:180-7.

37 Theodossiadis GP, Theodossiadis PG. The macular buckling procedure in the treatment of retinal detachment in highly myopic eyes with macular hole and posterior staphyloma: mean follow-up of 15 years. Retina 2005;25:285-9.

38 Sasoh M, Yoshida S, Ito Y, et al. Macular buckling for retinal detachment due to macular hole in highly myopic eyes with posterior staphyloma. Retina 2000;20:445-9.

39 Mateo C, Burés-Jelstrup A, Navarro R, et al. Macular buckling for eyes with myopic foveoschisis secondary to posterior staphyloma. Retina 2012;32:1121-8.

40 Schepens CL, Okamura ID, Brockhurst RJ. The scleral buckling procedures. I. Surgical techniques and management AMA Arch Ophthalmol 1957:58:797-811.

41 Klöti R. Silver clip for central retinal detachments with macular hole. Mod Probl Ophthalmol 1974;12:330-6.

42 Rosengren $B$. The silver plomb method in amotio retinae: clinical experience and results. Bib/ Ophthalmol 1966;70:253-6.

43 Siam AL. Management of central retinal detachment due to a macular hole. Br J Ophthalmol 1973;57:351-4.

44 Mortada A. Surgery of giant horse-shoe retinal tear extending behind the equator of the globe by an oblique encircling silastic $3 \mathrm{~mm}$ band passing posterior to the tear without evacuation of the subretinal fluid. Bull Ophthalmol Soc Egypt 1974:67:151-7

45 Parolini B, Frisina R, Pinackatt $S$, et al. A new I-shaped design of macular buckle to support a posterior staphyloma in high myopia. Retina 2013;33:1466-70.

46 Shen Z, Zhang Z, Zhang L, et al. Posterior scleral reinforcement combined with patching therapy for pre-school children with unilateral high myopia. Graefes Arch Clin Exp Ophthalmol 2015:253:1391-5. 